\title{
Application of Geographic Information System to control dengue outbreak in Tainan city, Taiwan 2015
} \author{
Chao-Yen Wu ${ }^{1, a}$, SHENG-ZHE LIN ${ }^{2, b}$, Chueh-Hui Yeh*3,c \\ 1,Department of Information Management, I-Shou University, Taiwan \\ ${ }^{2}$ Department of Health, Tainan City Government, \\ ${ }^{3}$ Department of Information Engineering, I-Shou University, Taiwan

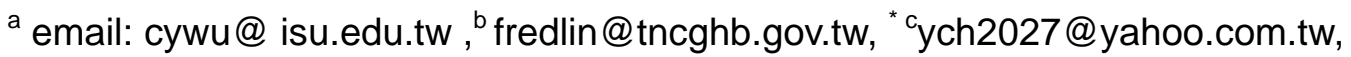

Keywords: Geographic Information System; dengue outbreak; Taiwan.

\begin{abstract}
Dengue is the most important arthropod-borne disease of human in the world. It is circulating in tropical and sub-tropical countries in Asia, Africa and the Americans. Taiwan has been also the affected area, and hundreds of people are infected over the past years mostly due to the transmission from imported cases. However, people infected dengue virus are over twenty thousand cases in a big history-record outbreak occurred in the southern Taiwan-Tainan city in 2015 because of the abundant rainfall and global warming which facilitated mosquito growth and virus spread. The Tainan dengue control team effectively controls scientific strategy and the control measures will constitute as a model worldwide.
\end{abstract}

\section{Introduction}

Dengue is the most important mosquito-borne viral infection worldwide, over 2.5 billion people are at risk of infection. Dengue virus has four serotypes because of gene sequence, and they spread in tropical and sub-tropical countries in Asia, Africa and the Americans. Besides, Aedesalbopivtus and Aedesaegyptiare two known dengue viral vector, people will be infected because of the virus-carried mosquito bites. Typically, most of people infected with denguevirus are asymptomatic or have only mild symptoms, but some cases may develop severe symptoms such as hemorrhaged or shock. There is no vaccine available preventing for defensing against dengue infection, so dengue still carries a health and financial burden in the affected area.Tainan city in southern Taiwan has been also the sporadically affected area, and only hundreds of people are infected over the past years mostly due to the imported cases from southeastern countries. However, in 2015, torrential rains and typhoons brought abundant rainfall in summer, offering appropriate environment for mosquito growth. Additionally, global warming caused the high temperature in whole year, providing a suitable condition for mosquito growth and virus spread. Therefore, history-record high dengue outbreak occurred in 2015 a total of over twenty thousand people were infected in the whole year. Starting from early September, a scientific control strategy was organized to apply real time GIS which integrate Distribution of cases.

\section{Literature Review}

\section{Geographic Information System}

Geographic Information Systems (GIS) is a computer-based tool that analyzes, stores,manipulates and visualizes geographic information on a map.Prediction of urban growth is very important for development planning and policy making as well as for urban management. In this model, five different but equal influential factors of urban expansion choices have been integrated.Debnath, Ripan and Amin, ATMNapplied thegeographic information system-based logical urban growth model for predicting spatial growth of an urban area[1]. Many reaches used the tracking Vaccination Teams During Polio Campaigns in Northern Nigeria by Use of Geographic Information System Technology[2]. Barcus, H. R. and Smith, L. J using Geographic Information Systems to Improve Practitioner Travel Efficiency in Home Based Primary Care[3].Ferguson, W. 
J.Kemp, K.Kost, G. using a geographic information system to enhance patient access to point-of-care diagnostics in a limited-resource setting[4]. Edward, J.Wang, S. S using Geographic Information Systems to Conduct a Needs Analysis to Develop Targeted Health Service Interventions for Underserved Hispanic Populations in Massachusetts[5].

\section{Dengue Outbreak}

Outbreaks have intensified in nearly all tropical areas, with endemic extent, deaths, and caseloads all reporting new highs, with the Americas reporting a whopping 2 million symptomatic cases. In tropical/subtropical Asia, nearly all nations had reported explosive increases. With one sole exception, China's caseload has plunged to less than a tenth of the prior year due to massive releases of sterilized mosquitos.

Dengue severity associated with age and a new lineage of dengue virus-type 2 during an outbreak[6].Dengue is an acute febrile illness caused by any of four dengue virus-types (DENV-14), which are transmitted by mosquitos of the genus Aedes and are endemic throughout the tropics and subtropics[7]. During 2010, an estimated 390 million DENV infections and 96 million clinically apparent cases occurred worldwide. Since 2005, two reported dengue outbreaks in Mexico that spread to Texas along the U.S.-Mexico border region have been reported[8]. Repetitive dengue outbreaks in East Africa: A proposed phased mitigation approach may reduce its impact[9]. Imported dengue from 2013 Angola outbreak: Not just serotype 1 was detected[10].

\section{Conclusion:}

Although this epidemic of dengue was record high in Tainan, Taiwan, our team still make great efforts and use unique scientific strategies to control the big outbreak effectively in 3-4 weeks. This lesson we learned in 2015 will become a model measure for dengue control worldwide.GIS applications can be effectively assisted epidemic decisions.This experiencein 2015 as a strategy to control dengue auxiliary model reference.Furthermore, the outbreak of dengue fever epidemic prevention strategy also include used NS1 rapid test for disease surveillance, marking early definite diagnosis and reporting. We integrated the all levels of medical resource including academic medical centers, metropolitan hospitals, local community hospitals and clinics, providing appropriate medical service for dengue patients. The Geographic information system and zonal policy for chemical spraying were unique strategies to control the epidemic of dengue rapidly and effectively.

\section{References}

[1] N. Raaber, I. Duvald, I. Riddervold, E. F. Christensen, and H. Kirkegaard, "Geographic information system data from ambulances applied in the emergency department: effects on patient reception," Scandinavian Journal of Trauma Resuscitation \& Emergency Medicine, vol. 24, Mar 2016.

[2] K. Touray, P. Mkanda, S. G. Tegegn, P. Nsubuga, T. B. Erbeto, R. Banda, et al., "Tracking Vaccination Teams During Polio Campaigns in Northern Nigeria by Use of Geographic Information System Technology: 2013-2015," Journal of Infectious Diseases, vol. 213, pp. S67-S72, May 2016.

[3] H. R. Barcus and L. J. Smith, "Facilitating native land reacquisition in the rural USA through collaborative research and Geographic Information Systems," Geographical Research, vol. 54, pp. 118-128, May 2016.

[4] W. J. Ferguson, K. Kemp, and G. Kost, "Using a geographic information system to enhance patient access to point-of-care diagnostics in a limited-resource setting," International Journal of Health Geographics, vol. 15, p. 12, Mar 2016.

[5] J. Edward and S. S. Wang, "Using Geographic Information Systems to Conduct a Needs Analysis to Develop Targeted Health Service Interventions for Underserved Hispanic Populations in 
Massachusetts," Nursing Research, vol. 65, pp. E110-E110, Mar-Apr 2016.

[6] F. Vairo, L. E. G. Mboera, P. De Nardo, N. M. Oriyo, S. Meschi, S. F. Rumisha, et al., "Clinical, Virologic, and Epidemiologic Characteristics of Dengue Outbreak, Dar es Salaam, Tanzania, 2014," Emerging Infectious Diseases, vol. 22, pp. 895-899, May 2016.

[7] P. C. G. Nunes, S. A. F. Sampaio, N. R. da Costa, M. C. L. de Mendonca, M. D. Q. Lima, S. E. M. Araujo, et al., "Dengue severity associated with age and a new lineage of dengue virus-type 2 during an outbreak in Rio De Janeiro, Brazil," Journal of Medical Virology, vol. 88, pp. 1130-1136, Jul 2016.

[8] M. Suleman, R. Faryal, U. B. Aamir, M. M. Alam, N. Nisar, S. Sharif, et al., "Dengue outbreak in Swat and Mansehra, Pakistan 2013; an epidemiological and diagnostic perspective," Asian Pacific Journal of Tropical Medicine, vol. 9, pp. 371-375, Apr 2016.

[9] M. Baba, J. Villinger, and D. K. Masiga, "Repetitive dengue outbreaks in East Africa: A proposed phased mitigation approach may reduce its impact," Reviews in Medical Virology, vol. 26, pp. 183-196, May 2016.

[10] C. Abreu, A. Silva-Pinto, D. Lazzara, J. Sobrinho-Simoes, J. T. Guimaraes, and A. Sarmentoa, "Imported dengue from 2013 Angola outbreak: Not just serotype 1 was detected," Journal of Clinical Virology, vol. 79, pp. 77-79, Jun 2016. 